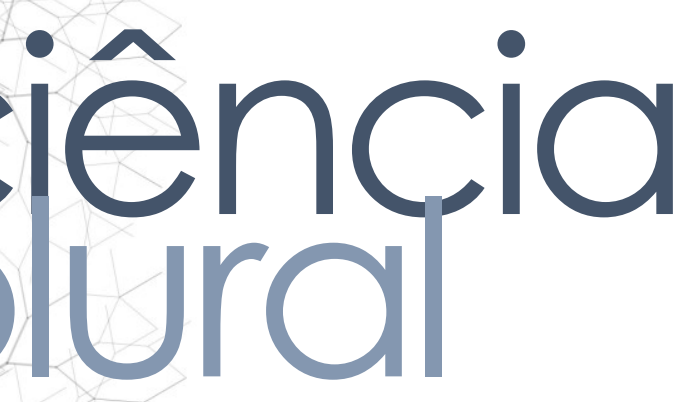

\title{
PREVALÊNCIA DA DOENÇA PERIODONTAL EM GESTANTES DE UMA UNIDADE BÁSICA DE SAÚDE EM NATAL/RN
}

Prevalence of periodontal disease in pregnant in a Basic Health Unit in Natal/RN

Prevalencia de enfermedades periodontales en mujeres embarazadas en una unidad de salud básica en Natal/RN

Nathália Brito da Costa $\bullet$ Cirurgiã-Dentista pela Universidade Federal do Rio Grande do Norte • E-mail: nathi_britto@hotmail.com

Edna Maria da Silva $\bullet$ Professora titular do Departamento de Odontologia da UFRN• E-mail: edna@reitoria.ufrn.br

Autora responsável pela correspondência:

Nathália Brito da Costa • E-mail: nathi_britto@hotmail.com 


\section{RESUMO}

Introdução: Durante a gravidez, os hormônios sexuais, estrógeno e progesterona, aumentam significativamente no organismo da mulher e parecem exercer efeitos marcantes e potencialmente prejudiciais sobre o metabolismo tecidual sendo, portanto, um fator agravante para o aumento da incidência da doença periodontal nesse período, assim como para o aumento da severidade da doença quando já instalada. Além disso, durante o período de gestação, ocorrem alterações nos níveis hormonais modificando as chances da doença periodontal se instalar ou se agravar. Objetivo: Estudar a prevalência e a severidade da doença periodontal, a identificação de dieta, higiene bucal e levantamento de lesões brancas ativas de esmalte nas gestantes cadastradas no Programa Pré-natal em uma Unidade Básica de Saúde em Natal/RN. Método: O estudo se caracteriza como seccional, descritivo e observacional, tendo como público alvo as gestantes cadastradas no programa de Prénatal da Unidade de Saúde de Aparecida. Foram incluídas 30 gestantes em estágios diversos de gravidez. Para a análise das condições periodontais utilizou-se Índice Periodontal Comunitário. Os dados obtidos foram tabulados em planilha Excel, a partir dos quais foram calculadas médias e os respectivos desvios-padrão. Além disso foi aplicado um questionário com questões semiestruturadas para a identificação de dieta, higiene bucal e levantamento de lesões brancas ativas de esmalte. Resultados: Constatou-se que metade das gestantes adotavam dieta rica em carboidratos, e de um modo geral, apresentavam higiene bucal insatisfatória; presença de lesão branca de esmalte ativa na maioria das gestantes examinadas; a condição periodontal avaliada por meio do índice escolhido que evidenciou alta prevalência de cálculo dentário, sangramento gengival e bolsas periodontais entre os sextantes examinados. Conclusões: De forma geral, as gestantes apresentaram uma condição de saúde bucal precária, com altas médias dos componentes do índice usado e presença de lesões brancas ativas de esmalte.

Palavras-Chave: Doença periodontal. Gravidez. Hormônios sexuais.

\section{ABSTRACT}

Introduction: During pregnancy, the sex hormones, estrogen and progesterone, increase significantly in the woman's body and seem to exert significant and potentially harmful effects on the tissue metabolism and, therefore, an aggravating factor to increase the incidence of periodontal disease in this period, as well as to increase the severity of the disease when already installed. In addition, during the gestation period, changes in hormone levels occur, modifying the chances of periodontal disease if it installs or worsens. Objective: To study the prevalence and severity of periodontal disease, identification of diet, oral hygiene and survey of active White enamel lesions among pregnant women enrolled in the Pré-natal Program in a asic Health Unit in Natal/RN. Methods: The study is characterized as sectional, escriptive and observational, with the target group of pregnant women enrolled in Pré-natal Program of the Aparecida Health Unit. Thirty pregnant women were uded in various stages of pregnancy. Periodontal Community Index was used to alyze the periodontal conditions. The data obtained were tabulated in Excel eadsheet, from this mean and the respective standard deviations were calculated. 
In addition, a questionnaire with semistructured questions was applied to the identification of diet, oral hygiene and survey of active white lesions of enamel. Results: It was found that half of the pregnant women adopted a diet rich in carbohydrates, and, in general, presented poor oral hygiene; presence of active enamel white lesion in the majority of pregnant women examined; the periodontal condition evaluated through of the showed a high prevalence of dental calculus, gingival bleeding and periodontal pockets among the sextants examined. Conclusions: In general, the pregnant women presented a precarious oral health condition, with high mean values of the components of the used index and the presence of active enamel white lesions.

Keywords: Periodontal disease. Pregnancy. Hormones.

\section{RESUMEN}

Introducción: durante el embarazo, las hormonas sexuales, el estrógeno y la progesterona, aumentan significativamente en el cuerpo de la mujer y parecen tener efectos marcados y potencialmente dañinos en el metabolismo de los tejidos, por lo que son un factor agravante de la mayor incidencia de enfermedad periodontal en este período. Así como para aumentar la gravedad de la enfermedad cuando ya está instalada. Además, durante el período de gestación, se producen cambios en los niveles hormonales, lo que cambia las posibilidades de que la enfermedad periodontal se estabilice o empeore. Objetivo: Estudiar la prevalencia y la gravedad de la enfermedad periodontal, identificación de la dieta, higiene bucal y estudio de lesiones activas del esmalte blanco en mujeres embarazadas registradas en el Programa Prénatal en una Unidad de Salud Básica en Natal/RN. Método: El estudio se caracteriza por ser descriptivo, descriptivo y observacional, dirigido a mujeres embarazadas registradas en el programa Pré-natal de la Unidad de Salud de Aparecida. Se incluyeron treinta mujeres embarazadas en diferentes etapas del embarazo. Para el análisis de las condiciones periodontales, se utilizó un índice periodontal comunitario. Los datos obtenidos se tabularon en una hoja de cálculo Excel, a partir de la cual se calcularon los promedios y las respectivas desviaciones estándar. Además, se aplicó un cuestionario con preguntas semiestructuradas para identificar la dieta, la higiene bucal y la encuesta de las lesiones activas del esmalte blanco. Resultados: encontró que la mitad de las mujeres embarazadas adoptaron una dieta rica en carbohidratos y, en general, presentaron una higiene bucal insatisfactoria; presencia de lesión del esmalte blanco activa en la mayoría de las embarazadas examinadas; La condición periodontal evaluada mediante el índice mostró una alta prevalencia de cálculo dental, sangrado gingival y bolsas periodontales entre los sextantes examinados. Conclusiones: en general, las mujeres embarazadas presentaron una condición de salud bucal precaria, con promedios altos de los componentes del índice utilizado y la presencia de lesiones activas de esmalte blanco.

labras clave: Enfermedad periodontal. Embarazo. Hormonas sexuales. 


\section{Introdução}

Dentre as diversas patologias que acometem o ambiente bucal, a doença periodontal se destaca por sua alta prevalência. Esta doença caracteriza-se como uma doença infecciosa inflamatória, que acomete tanto os tecidos de proteção como os tecidos de sustentação do periodonto. Sabe-se, na atualidade, que a presença de biofilme dentário, constituído por depósitos bacterianos e constituintes salivares, está diretamente ligada a instalação e progressão da doença periodontal, tornando-a uma doença biofilme dependente ${ }^{1}$.

Quando há um desequilíbrio entre a agressão microbiana e a resposta do hospedeiro, a doença periodontal pode ser instalada. Essa resposta se manifesta como sendo uma gengivite e, quando não tratada, a sua progressão pode evoluir para uma periodontite ${ }^{2}$.

A predisposição ao desenvolvimento da doença periodontal também depende da associação com outros fatores, como a condição socioeconômica, o acesso a serviços de saúde bucal, a presença de doenças sistêmicas, a alterações hormonais, dentre outros ${ }^{3}$.

Em função dos hormônios estrógeno e progesterona serem predominantes no organismo da mulher, estas são mais sensíveis às alterações hormonais que os homens. Tanto o estrógeno quanto a progesterona aumentam significativamente no organismo feminino no período da gravidez. As evidências científicas indicam que nessa fase a mulher fica sujeita a efeitos prejudiciais sobre o metabolismo tecidual. Dentre esses efeitos adversos tem-se o aumento da incidência da doença periodontal, assim como também o incremento da severidade da doença quando já instalada.2

Assim, a resposta inflamatória nos tecidos periodontais, frente à presença de biofilme dentário, é exacerbada devido aos elevados níveis de hormônios sexuais durante a gestação. Daí decorre a necessidade de acompanhamento das gestantes por rofissionais da Odontologia na perspectiva do atendimento clínico com vistas ao gnóstico e tratamento adequado, além da institucionalização de atividades de entação para higiene bucal e controle do biofilme, como forma de controle das iodontopatias. 
Considerando-se a escassez de estudos que correlacionem a gestação e a doença periodontal em função do trimestre gestacional, justifica-se a realização de estudos com a finalidade de observar se há alteração da condição periodontal durante o período gestacional. Dessa forma, poderá ser estabelecida uma adequada assistência à gestante em programas de acompanhamento pré-natal, principalmente, no âmbito do Sistema Único de Saúde (SUS).

Portanto, este trabalho tem como objetivo estudar a prevalência e a severidade da doença periodontal nas gestantes cadastradas no Programa Pré-natal em uma Unidade Básica de Saúde em Natal/RN.

\section{Metodologia}

A pesquisa consiste em um estudo seccional, observacional e descritivo realizado com todas as gestantes participantes do programa de pré-natal em uma Unidade Básica de Saúde (UBS), em Natal-RN, que estavam de acordo com os critérios de inclusão apresentados neste estudo.

Essas gestantes foram distribuídas em função do trimestre gestacional como: Grupo I (GI) = 6 grávidas no primeiro trimestre, Grupo II (GII) = 11 grávidas do segundo trimestre e Grupo III (GIII) = 13 grávidas no terceiro trimestre, perfazendo um total de 30 grávidas. Foram realizados três levantamentos, correspondentes a cada grupo, na mesma época, a partir dos quais foram calculadas as médias dos escores do Índice Periodontal Comunitário (CPI ), segundo seus constituintes: cálculo, sangramento gengival e bolsas. Assim todas as gestantes foram submetidas a um atendimento clínico, no qual foi realizado um diagnóstico inicial da condição periodontal (Apêndice B).

O Índice Periodontal Comunitário permite avaliar a condição periodontal quanto à higidez, sangramento e presença de cálculo ou bolsa. Para a realização do xame foi necessária a utilização de uma sonda específica (Sonda OMS), espelho bucal equipamento de proteção individual completo. Para isso, a boca foi dividida em tantes definidos pelos dentes: 18-14, 13-23, 24-28, 38-34, 33-43 e 44-48. A presença dois ou mais dentes sem indicação de exodontia é pré-requisito ao exame do 
sextante. Sem isso, o sextante é excluído. O exame foi feito nos dentes índices de cada sextante $(16 / 17,11 / 21,26 / 27,36 / 37,31 / 41,46 / 47)$ e embora 10 dentes sejam examinados, apenas 6 anotações são feitas: uma por sextante, relativa à pior situação diagnosticada, tendo sido adotados os seguintes códigos:

0 - sextante hígido;

1 - sextante com sangramento (observado diretamente ou com espelho, após sondagem);

2 - cálculo (qualquer quantidade, mas com toda a área preta da sonda visível);

3 - bolsa de $4 \mathrm{~mm}$ a $5 \mathrm{~mm}$ (margem gengival na área preta da sonda);

4 - bolsa de 6 mm ou mais (área preta da sonda não está visível);

X - sextante excluído (menos de 2 dentes presentes);

9 - sextante não examinado.

Além da condição periodontal, foram avaliados a higiene bucal e o tipo de dieta (cariogênica e não cariogênica). A dieta cariogênica é considerada rica em alimentos de grande teor de açúcar, como refrigerantes, chocolates, balas e biscoitos. A higiene bucal foi classificada como satisfatória ou insatisfatória em função do acúmulo de biofilme e a presença de lesões brancas de esmalte ativas, assim consideradas quando apresentavam a superfície rugosa e opaca em regiões de retenção de biofilme, como no terço cervical, proximal e oclusal dos dentes.14 Para a identificação do nível socioeconômico, utilizou-se a seguinte divisão de classes: A (R\$ 18.740,01 ou mais), B ( $\mathrm{R} \$ 9.370,01$ a $\mathrm{R} \$ 18.740,00), \mathrm{C}(\mathrm{R} \$ 3.748,01$ a $\mathrm{R} \$ 9.370,00), \mathrm{D}(\mathrm{R} \$$ $1.874,01$ a $\mathrm{R} \$ 3.748,00)$ e E (até $\mathrm{R} \$ 1.874,00) .15$

A execução da pesquisa seguiu a orientação da Resolução 466/2012-CNS, que garante o respeito à dignidade humana e exige que toda pesquisa se processe com consentimento livre e esclarecido dos participantes, indivíduos ou grupos que, por si ou por seus representantes legais, manifestem a sua anuência à participação na esquisa. Portanto, a pesquisa foi aprovada pelo Comitê de Ética em Pesquisa da RN, por meio da Plataforma Brasil, com parecer número 2.763.807. Dessa forma, as as participantes da pesquisa assinaram o Termo de Consentimento Livre e 
Esclarecido - TCLE (Apêndice A), estando, portanto, de acordo com a participação no estudo.

Os dados obtidos foram inseridos em uma planilha Excel a partir da qual foram calculadas as médias e desvios-padrão referentes ao Índice CPI, presença de lesão branca ativa do esmalte, além da higiene bucal e da dieta.

Foram incluídas no estudo todas as gestantes integrantes do programa pré-natal em uma UBS em Natal/RN, maiores de dezoito anos, que não eram portadoras de doenças sistêmicas associadas a gestação e que concordaram em participar da pesquisa, assinando o Termo de Consentimento Livre e Esclarecido-TCLE. Foram excluídas as gestantes não cadastradas no programa de pré-natal da referida unidade de saúde, menores de dezoito anos, que eram portadoras de doenças sistêmicas associadas a gestação e que não preencheram o Termo de Consentimento Livre e Esclarecido, por não concordarem com a participação.

\section{Resultados e Discussão}

Os dados referentes à idade, nível de escolaridade, condição socioeconômica e o tempo de gestação estão apresentados na Tabela 1. As gestantes tinham idade média de 27,4 anos e foram distribuídas nas faixas etárias: 18-24, 25-35 e 36-40 anos. Quanto à escolaridade, observou-se que $13,3 \%$ eram analfabetas ou tinham ensino fundamental incompleto e que $86,7 \%$ cursaram o ensino fundamental completo ou mais. Em relação ao nível socioeconômico, verificou-se que a distribuição das gestantes de acordo com as classes sociais demonstra a precariedade financeira do grupo: A $(0 \%), \mathrm{B}(0 \%), \mathrm{C}(0 \%), \mathrm{D}(26,7 \%)$ e $\mathrm{E}(73,3 \%)$. Além disso, observou-se que $20 \%$ estavam no primeiro trimestre gestacional, $36,7 \%$ no segundo trimestre e $43,3 \%$ no terceiro trimestre.

Com isso, pode-se observar que a maior parte dessas mulheres estão em ituação de pobreza, mas possuem um nível de estudo que as permite ter noção da mportância dos cuidados com a saúde como um todo e da saúde bucal, em particular. sim, esperava-se que as mesmas possuíssem bons índices de higiene oral e, sequentemente, melhores condições de saúde bucal. 


\section{ciência plural}

Tabela 1 - Distribuição das gestantes em relação à idade, escolaridade, nível socioeconômico e trimestre gestacional. Natal, RN, 2019.

\begin{tabular}{|c|c|c|}
\hline \multirow{3}{*}{ Idade $(\%)$} & $18-24$ anos & $40 \%$ \\
\hline & $25-35$ anos & $43,3 \%$ \\
\hline & $36-40$ anos & $16,7 \%$ \\
\hline \multirow[t]{2}{*}{ Escolaridade (\%) } & Analfabeto ou Ens. Fund. Incompleto & $13,3 \%$ \\
\hline & Ens. Fund. Completo ou Ens. Médio Completo & $86,7 \%$ \\
\hline \multirow{5}{*}{ Nível Socioeconômico (\%) } & A & $0 \%$ \\
\hline & $\mathrm{B}$ & $0 \%$ \\
\hline & $\mathrm{C}$ & $0 \%$ \\
\hline & $\mathrm{D}$ & $26,7 \%$ \\
\hline & $\mathrm{E}$ & $73,3 \%$ \\
\hline \multirow{3}{*}{ Trimestre Gestacional (\%) } & $1^{\circ}$ & $20 \%$ \\
\hline & $2^{\circ}$ & $36,7 \%$ \\
\hline & $3^{\circ}$ & $43,3 \%$ \\
\hline
\end{tabular}

Fonte: Autor (2019).

A condição de saúde bucal foi analisada em função da dieta (cariogênica ou não cariogênica), higiene oral (satisfatório ou insatisfatório), lesão branca ativa (presente ou ausente) e CPI (presença de cálculo dentário, sangramento gengival, bolsa periodontal com profundidade de sondagem de 4 a $5 \mathrm{~mm}$ e bolsa periodontal com profundidade de sondagem de $6 \mathrm{~mm}$ ou mais). Esses dados podem ser observados na abela 2 
Tabela 2 - Dados referentes à dieta, higiene oral, lesão branca ativa e índice CPI, em relação ao trimestre gestacional. Natal, RN, 2019.

\begin{tabular}{|c|c|c|c|c|}
\hline \multirow{10}{*}{$1^{\circ}$ Trimestre } & \multirow[t]{2}{*}{ Dieta $(\%)$} & Cariogênica & $50,0 \%$ & \\
\hline & & $\begin{array}{l}\text { Não } \\
\text { cariogênica }\end{array}$ & $50,0 \%$ & \\
\hline & \multirow{2}{*}{$\begin{array}{l}\text { Higiene Oral } \\
(\%)\end{array}$} & Insatisfatório & $83,3 \%$ & \\
\hline & & Satisfatório & $16,7 \%$ & \\
\hline & \multirow{2}{*}{$\begin{array}{l}\text { Lesão Branca } \\
\text { Ativa (\%) }\end{array}$} & Presente & $83,3 \%$ & \\
\hline & & Ausente & $16,7 \%$ & \\
\hline & \multirow{4}{*}{ CPI (sextante) } & Cálculo & $\bar{X}=4,0$ & $\mathrm{DP}=1,6$ \\
\hline & & Sangramento & $\bar{X}=2,7$ & $\mathrm{DP}=1,4$ \\
\hline & & Bolsa 4 -5 mm & $\bar{X}=2,2$ & $\mathrm{DP}=1,3$ \\
\hline & & $\begin{array}{l}\text { Bolsa } 6 \mathrm{~mm} \text { ou } \\
\text { mais }\end{array}$ & $\bar{X}=0,7$ & $\mathrm{DP}=1,5$ \\
\hline \multirow{10}{*}{$2^{\circ}$ Trimestre } & \multirow[t]{2}{*}{ Dieta (\%) } & Cariogênica & $45,5 \%$ & \\
\hline & & $\begin{array}{l}\text { Não } \\
\text { cariogênica }\end{array}$ & $54,5 \%$ & \\
\hline & \multirow{2}{*}{$\begin{array}{l}\text { Higiene Oral } \\
(\%)\end{array}$} & Insatisfatório & $81,8 \%$ & \\
\hline & & Satisfatório & $18,2 \%$ & \\
\hline & \multirow{2}{*}{$\begin{array}{l}\text { Lesão Branca } \\
\text { Ativa (\%) }\end{array}$} & Presente & $63,6 \%$ & \\
\hline & & Ausente & $36,4 \%$ & \\
\hline & \multirow{4}{*}{ CPI (sextante) } & Cálculo & $\bar{X}=4,2$ & $\mathrm{DP}=1,7$ \\
\hline & & Sangramento & $\bar{X}=3,8$ & $\mathrm{DP}=1,1$ \\
\hline & & Bolsa $4-5 \mathrm{~mm}$ & $\bar{X}=2,3$ & $\mathrm{DP}=1,1$ \\
\hline & & $\begin{array}{l}\text { Bolsa } 6 \mathrm{~mm} \text { ou } \\
\text { mais }\end{array}$ & $\bar{X}=0,2$ & $\mathrm{DP}=0,6$ \\
\hline \multirow{10}{*}{$3^{\circ}$ Trimestre } & \multirow[t]{2}{*}{ Dieta (\%) } & Cariogênica & $53,8 \%$ & \\
\hline & & $\begin{array}{l}\text { Não } \\
\text { cariogênica }\end{array}$ & $46,2 \%$ & \\
\hline & \multirow{2}{*}{$\begin{array}{l}\text { Higiene Oral } \\
(\%)\end{array}$} & Insatisfatório & $61,5 \%$ & \\
\hline & & Satisfatório & $38,5 \%$ & \\
\hline & \multirow{2}{*}{$\begin{array}{l}\text { Lesão Branca } \\
\text { Ativa (\%) }\end{array}$} & Presente & $46,2 \%$ & \\
\hline & & Ausente & $53,8 \%$ & \\
\hline & \multirow{4}{*}{ CPI (sextante) } & Cálculo & $\bar{X}=3,8$ & $\mathrm{DP}=1,2$ \\
\hline & & Sangramento & $\bar{X}=2,3$ & $\mathrm{DP}=1,0$ \\
\hline & & Bolsa 4-5 mm & $\bar{X}=1,1$ & $\mathrm{DP}=1,2$ \\
\hline & & $\begin{array}{l}\text { Bolsa } 6 \mathrm{~mm} \text { ou } \\
\text { mais }\end{array}$ & $\bar{X}=0,3$ & $\mathrm{DP}=0,7$ \\
\hline
\end{tabular}

$=$ Média; DP $=$ Desvio Padrão.

hte: Autor (2019). 
Ao analisar os dados referentes à dieta de cada paciente, a partir do consumo de alimentos cariogênicos ou de alto teor de açúcar, como refrigerantes, chocolates, balas, biscoitos, entre outros, verificou-se que nos três períodos gravídicos estudados, as gestantes apresentaram comportamentos semelhantes, ou seja, em torno da metade respondeu adotar o consumo de alimentos ricos em açúcares e a outra metade negou esse consumo. Dados da literatura indicam que no período gestacional, aumenta-se a frequência com que as gestantes se alimentam, inclusive com alimentos de potencial cariogênico.16

A cárie, sendo uma doença multifatorial, é influenciada por fatores como composição e frequência da dieta, capacidade tampão da saliva, fatores socioeconômicos, educacionais e comportamentais. Quanto à dieta, já está comprovado há muito tempo pelas evidências científicas que os carboidratos são fermentados pelas bactérias presentes no biofilme dentário, resultando na produção de ácidos que geram uma desmineralização da superfície dentária. Além disso, esse biofilme vai ficando cada vez mais complexo, com menor teor de oxigênio, levando, assim, a uma sucessão microbiana, tornando-o patogênico para o periodonto também ${ }^{17}$.

Quanto à higiene bucal, observou-se que as gestantes, de um modo geral, apresentaram precária higiene bucal, independente do trimestre de gestação, denotando pouca valorização às medidas de prevenção e controle das doenças biofilme dependentes, fato que poderia levar a um agravamento da condição periodontal durante a gravidez. No tocante ao atendimento odontológico, é importante ressaltar que o biofilme dentário, podendo localizar-se supra ou subgengivalmente, é fator etiológico primário no desenvolvimento da doença periodontal. Isso ocorre, pois há um desequilíbrio entre a agressão provocada pelo biofilme e a resposta inflamatória e imune do hospedeiro ${ }^{2}$. Dessa forma, é importante uma higiene oral satisfatória, de forma que o biofilme dentário seja desorganizado e não interfira na saúde do periodonto.

Por outro lado, ao analisar a presença de lesão branca de esmalte ativa, servou-se uma prevalência significativa, possivelmente, em consequência do 
consumo de alimentos com alto potencial cariogênico associado à negligência com a remoção do biofilme dentário. Essas condições estão explícitas na Tabela 2, que permite uma análise do conjunto das variáveis estudadas.

As lesões brancas indicam a presença da cárie e também a configuração de risco. Essas lesões podem se apresentar na forma ativa ou inativa em regiões de retenção de biofilme, como no terço cervical, proximal e oclusal dos dentes. Quando ativas, as lesões se apresentam com a superfície rugosa e opaca, enquanto que quando inativas, apresentam-se com a superfície lisa e brilhante. Tratamentos não invasivos, como a aplicação tópica de flúor, permitem a remineralização do esmalte dentário, tornando as lesões brancas ativas em lesões inativas.14 Portanto, é de grande importância a prevenção dessas lesões com o controle do biofilme dentário e uma dieta não cariogênica, além da institucionalização do processo de reversão a fim de a progressão da cárie seja controlada.

Quanto à condição periodontal, analisada a partir dos componentes do índice $\mathrm{CPI}$, analisando-se separadamente a presença de cálculo dentário, verificou-se que este estava, de forma geral, presente em 4 de cada 6 sextantes examinados. Este dado sugere a falta de acesso dessas mulheres ao serviço odontológico e hábitos de higiene oral inadequados, mesmo antes da condição de gravidez, assim como a ausência de tratamento por ocasião do pré-natal. O cálculo dentário é um fator retentivo de biofilme, e como já foi visto nesse estudo, o biofilme dentário é um fator etiológico primário para o desenvolvimento da doença periodontal. Além disso, ele interfere nos processos de desmineralização e remineralização do esmalte, podendo gerar a cárie ${ }^{14}$. Portanto, pode-se perceber a necessidade de adequação do meio bucal dessas gestantes, iniciando com profilaxia dentária e raspagem e alisamento corono-radicular dos sextantes ${ }^{18}$.

O sangramento gengival, outro fator avaliado no CPI, também se comportou de forma semelhante nas gestantes, em todos os trimestres gestacionais, apresentando angramento em 3 a 4 sextantes de cada 6 sextantes examinados. O aumento nos níveis ormonais de estrógeno e progesterona durante a gravidez desencadeia uma série de ores que predispõem o desenvolvimento da doença periodontal, principalmente o 
sangramento gengival. No tecido gengival há receptores para esses hormônios que lá se acumulam e funcionam como fator de crescimento para as bactérias, pois estas os utilizam como nutrientes. Além disso, a elevação dos níveis hormonais modifica a resposta inflamatória e imunológica da gestante e influenciam na microvascularização, aumentando a permeabilidade vascular e a síntese de prostaglandinas. Dessa forma, durante a gravidez pode haver um aumento no índice de sangramento gengival devido aos fatores citados anteriormente. ${ }^{5}$

Por fim, ao avaliar a presença de bolsas periodontais, observou-se que não ocorreram variações significativas ao se comparar os trimestres gestacionais. A bolsa periodontal com profundidade de sondagem de $4 \mathrm{~mm}$ a $5 \mathrm{~mm}$ apresentou-se em média de 1 a 2 sextantes de cada 6 examinados. Já a bolsa periodontal com profundidade de sondagem de $6 \mathrm{~mm}$ ou mais esteve presente em menos de 1 sextante de cada 6 . As alterações hormonais interferem apenas no tecido gengival, influenciando numa resposta inflamatória mais exacerbada, não causando alterações na inserção periodontal. Esta é uma situação transitória, pois após o parto os níveis hormonais voltam aos valores normais deixando de interferir na condição periodontal ${ }^{6}$.

Tabela 3 - Relação entre os níveis de higiene bucal e os componentes do índice CPI. Natal, RN, 2019.

\begin{tabular}{llllllllll}
\hline & $\begin{array}{l}\text { Higiene } \\
\text { Bucal } \\
(\%)\end{array}$ & $\begin{array}{l}\text { Cálculo } \\
\text { (sextante) }\end{array}$ & \multicolumn{2}{c}{$\begin{array}{l}\text { Sangramento } \\
\text { (sextante) }\end{array}$} & $\begin{array}{l}\text { Bolsa 4-5 } \\
\text { mm } \\
\text { (sextante) }\end{array}$ & $\begin{array}{l}\text { Bolsa 6 ou } \\
\text { mais mm } \\
\text { (sextante) }\end{array}$ \\
\hline Insatisfatória & $73,3 \%$ & $\bar{X}$ & DP & $\bar{X}$ & DP & $\bar{X}$ & DP & $\bar{X}$ & DP \\
\hline Satisfatória & $26,7 \%$ & $\bar{X}$ & DP & $\bar{X}$ & DP & $\bar{X}$ & DP & $\bar{X}$ & DP \\
& & 2,4 & 1,2 & 3,0 & 1,5 & 1,7 & 1,4 & 0,3 & 0,9 \\
\hline
\end{tabular}

$=$ Média; $\mathrm{DP}=$ Desvio Padrão.

onte: Autor (2019).

A tabela 3 mostra uma relação entre a higiene bucal (insatisfatório e satisfatório) condição periodontal das grávidas independente do trimestre de gestação. 
Constatou-se que 73,3\% das gestantes apresentavam higiene insatisfatória, enquanto que 26,7\% apresentavam higiene satisfatória. Entre grávidas com higiene oral insatisfatória, o cálculo dentário estava presente em 4,4 sextantes a cada 6 sextantes; o sangramento gengival em 3 sextantes; a presença de bolsa periodontal de $4 \mathrm{~mm}$ a 5 mm de profundidade em 1,7 sextantes; e bolsa periodontal de $6 \mathrm{~mm}$ ou mais em 0,3 sextante. Quanto às gestantes com higiene oral satisfatória, o cálculo se apresentou em 2,9 sextantes; o sangramento em 2,6 sextantes; bolsa de $4 \mathrm{~mm}$ a $5 \mathrm{~mm}$ em 1,9 sextantes; e bolsa de $6 \mathrm{~mm}$ ou mais estava presente em 0,5 sextante. Dessa forma, pôde-se perceber que também não ocorreram diferenças de uma condição para a outra. A expectativa era que a remoção do biofilme pudesse contribuir para uma condição periodontal mais satisfatória, como já é conhecimento corrente na literatura. Conjectura-se que essas mulheres não tenham recebido a atenção adequada por ocasião do pré-natal.

Essas informações indicam que, de forma geral, não há uma boa saúde periodontal nessas mulheres. Portanto, há a necessidade de intervenção odontológica para se evitar a progressão da doença, sendo fundamental a remoção dos fatores retentivos de biofilme, assim como orientações de higiene bucal para a melhoria da situação diagnosticada.

Porém, a literatura mostra que há resistência por parte dos cirurgiões-dentistas em realizarem tratamento odontológico em gestantes, pois se sentem inseguros devido a uma abordagem deficiente sobre o tema durante a formação acadêmica. A insegurança também ocorre por parte das gestantes, pois muitas acreditam que o tratamento odontológico pode causar anormalidades congênitas ou aborto. Todavia, estudos mostram que as gestantes podem fazer o tratamento odontológico sem que ocorra malefícios a saúde do seu bebê, preferencialmente, com consultas durante o segundo trimestre gestacional ${ }^{18}$. Além disso, a inserção do cirurgião dentista no préatal odontológico, ora como disseminador de informações para a melhoria da ondição de saúde bucal da gestante, tem como consequência que essa mulher se torne ente multiplicadora de saúde e gere benefícios ao seu bebê19. 
Diante dos resultados obtidos e apresentados neste estudo, observa-se que não houve diferenças marcantes entre as condições de saúde das gestantes nos trimestres gestacionais, pois, no geral, os índices apontaram para uma condição precária da saúde bucal dessas mulheres. Além disso, é importante ressaltar que as consultas periódicas durante a gravidez são bastante relevantes para o controle e manutenção da saúde bucal, eliminando focos infecciosos e evitando a instalação e progressão de doenças por meio de intervenções preventivas.

\section{Conclusões}

A análise dos dados da pesquisa permite concluir que as gestantes apresentaram alta prevalência de problemas periodontais, possivelmente, em decorrência de uma higiene bucal insatisfatória e consumo de alimentos ricos em carboidratos fermentáveis.

Essa situação mostra uma deficiência no acompanhamento clínico dessas gestantes por ocasião do pré-natal, reafirmando a baixa resolutividade da assistência odontológica mesmo em programas especiais. O programa de pré-natal odontológico é de grande importância para melhorar a condição de saúde bucal das gestantes e, consequentemente, sua saúde de forma geral, o qual deve ser estimulado e normatizado na rede de serviço do Sistema Único de Saúde.

\section{Referências}

1 Newman MG, Takei H, Klokkevold PR, Carranza Júnior FA. Carranza Periodontia Clínica. 12th. Rio de Janeiro: Elsevier; 2016.

2 Santos JF, Pillon FL. A influência dos hormônios sexuais femininos sobre a manifestação clínica das doenças periodontais: Revisão de Literatura. Periodontia. 2009;19(3):34-40.

Almeida RF, Pinho MM, Lima C, Faria I, Santos P, Bordalo C. Associação entre ença periodontal e patologias sistêmicas. Rev Port Med Geral Fam. 2006;22(3):379- 
4 Lorenzoni BCD. Avaliação da doença periodontal na Unidade São Sebastião no município de Ribas do Rio Pardo no Mato Grosso do Sul [monografia]. Ribas do Rio Prado: Universidade Federal do Mato Grosso do Sul; 2011.

5 Bertolini PFR, Biondi Filho O, Niero BG, Saraceni CHC, Splendore SMG, Pomilio A, et al. Medicina periodontal e a mulher: a importância do seu conhecimento para uma abordagem preventiva por ginecologistas/obstetras e cirurgiões-dentistas. Rev Ciênc Méd. 2007;16(3):175-185.

6 Cunha DPR. Doença periodontal na gestação [monografia]. Porto Alegre: Universidade Federal do Rio Grande do Sul; 2011.

7 Louro PM, Fiori HH, Louro Filho P, Steibel J, Fiori RM. Doença Periodontal na gravidez e baixo peso ao nascer. J Pediatr. 2001;77(1):23-28.

8 Lindhe J, Karring T, Lang NP. Tratado de Periodontia Clínica e Implantodontia Oral. 5th. Rio de Janeiro: Guanabara Koogan; 2010.

9 Reis DM, Pitta DR, Ferreira HMB, Jesus MCP, Moraes MEL, Soares MG. Educação em saúde como estratégia de promoção de saúde bucal em gestantes. Ciênc saúde colet. 2010;15(1):269-276.

10 Scavuzzi AIF, D’Agostino ES, Cunha YMS, Guimarães ARD. Contribuição ao estudo da cárie dental e da doença periodontal durante a gestação na cidade de Feira de Santana, Bahia, Brasil. Pesq Bras Odontoped Clin Integr. 2010;10(3):351-356.

11 Tretin MS, Scortegagna SA, Dal'Bello MS, Bittencourt ME, Linden MSS, Viero R, et al. Doença periodontal em gestantes e fatores de risco para o parto prematuro. RFO. 2007;12(1):47-51.

12 Moimaz SAS, Carmo MP, Zina LG, Saliba NA. Associação entre condição periodontal de gestantes e variáveis maternas e de assistência à saúde. Pesqui Bras Odontopediatria Clin Integr. 2010;10(2):271-278.

13 Gusmão ES, Cimões R, Souza ACP, Silva ACCR, Santos MCAL, Santos RL. O PSR como meio de diagnóstico ambulatorial em gestantes. Int J Dent. 2007;6(4):108-112.

14 Barbosa BG, Silva VL, Gontijo IG, Andrade RMPMB, Nogueira RD, GeraldoMartins VR. Tratamento de lesão de mancha branca com infiltrante resinoso: relato de caso. Rev Odontol Bras Central. 2018;27(83):252-256.

Instituto Brasileiro de Geografia e Estatística (IBGE). Pesquisa Nacional por mostra de Domicílios. Brasília: IBGE; 2015.

Melo NSFO, Ronchi R, Mendes CS, Mazza VA. Hábitos alimentares e de higiene influenciando a saúde bucal da gestante. Cogitare Enferm. 2007;12(2):189-197. 


\section{ciência
purála}

17 Leites ACBR, Pinto MB, Sousa ER. Aspectos microbiológicos da cárie dental. Salusvita. 2006;25(2):239-252.

18 Poletto VC, Stona P, Weber JBB, Fritscher AMG. Atendimento odontológico em gestantes: uma revisão da literatura. Stomatos. 2008;14(26):64-75.

19 Lopes IKR, Pessoa DMV, Macêdo GL. Autopercepção do pré-natal odontológico pelas gestantes de uma unidade básica de saúde. Rev Ciênc Plu. 2018;4(2):60-72. 